American Journal of Biochemistry and Biotechnology 3 (4): 187-192, 2007

ISSN 1553-3468

(C) 2007 Science Publications

\title{
Suppression of Basal and Cytokine Induced Expression of MHC, ICAM 1 and B7 Markers on Mouse Lung Epithelial Cells Exposed to Diesel Exhaust Particles
}

\author{
${ }^{1,2}$ Rajiv K. Saxena, ${ }^{1}$ Wanda Williams and ${ }^{1}$ M. Ian Gilmour \\ ${ }^{1}$ Immunotoxicology Division, ETD, NHEERL \\ US Environmental Protection Agency, Research Triangle Park, NC \\ ${ }^{2}$ School of Life Sciences, Jawaharlal Nehru University, New Delhi
}

\begin{abstract}
Diesel exhaust particles (DEP) constitute a significant component of airborne particulates in urban environment. Exposure to DEP is known to enhance susceptibility to viral and bacterial infections. We hypothesized that DEP could partially exert its effect on disease susceptibility by interfering with antigen presentation required for the generation of protective adaptive immune responses. MHC class I and class II molecules, ICAM 1 (CD54) and B7.1 (CD80) are intimately involved with the presentation of viral and bacterial antigens to T cells. In the present study we examined the effect of in vitro exposure to DEP on basal and cytokine induced expression levels of these key molecules on mouse lung epithelial cells. LA4 and TC1 mouse epithelial cell lines were activated with a cytokine mixture comprising IFN $\gamma$, TNF $\alpha$ and IL1 $\beta$, in the presence or absence of DEP. CM significantly boosted the expression of MHC I and ICAM 1 antigens on epithelial cells. Exposure to DEP significantly inhibited the basal and CM induced expression of MHC I and ICAM 1 expression, but did not interfere with the constitutive expression of B7.1 on epithelial cells. Significant expression of MHC II antigens, which is needed for presentation of exogenous antigenic peptides, was not detected on either LA4 or TC1 cells. These results suggest that DEP may interfere with the expression of the cellular markers involved in the presentation of viral antigens.
\end{abstract}

Key words: MHC class I, ICAM 1, B7, lung epithelial cells, diesel exhaust particles, IFN $\gamma$, TNF $\alpha$

\section{INTRODUCTION}

Efficient transport of oxygen and $\mathrm{CO}_{2}$ across lung alveoli is vital for the sustenance of life and it is imperative that the epithelial surface be kept free from accumulation of airborne pathogens and pollutants that may induce acute inflammatory responses or even cause permanent damage, which can impede this process. Innate and adaptive immune mechanisms directed against airborne pathogens in the lung can result in altered pathology and play an important role in keeping the organ free from pathogens and disease. Adaptive immune responses require the presentation of microbe-derived peptides to $T$ cells. For viruses, antigen presenting cells must degrade viral proteins and associate them with class I MHC molecules whereas bacterial peptides are associated with class II MHC molecules expressed on the antigen presenting cells ${ }^{[1]}$. Besides MHC molecules, antigen presenting cells must also express a variety of other membrane proteins such as ICAM 1, B7.1, B7.2 that participate as accessory molecules in the interaction between $\mathrm{T}$ cells and the antigen presenting cells ${ }^{[2]}$. While all nucleated cells in the body including lung epithelial cells express class I MHC molecules and are capable of presenting viral antigens, only limited cell types like dendritic cells and macrophages have the ability to process and present bacterial antigens in the context of MHC II molecules ${ }^{[3,4]}$. Some evidence also supports the ability of lung epithelial cells to present pathogen derived peptides to T cells via the MHC I pathway ${ }^{[3-7]}$.

Factors that regulate the expression of $\mathrm{MHC}$ molecules and the accessory molecules involved in antigen presentation may determine the quality of immune response against pathogens. Members of the toll-like receptor (TLR) family, expressed on a variety of immune and non-immune cells rapidly sense the presence of pathogens in lungs. TLR activation, during early phases of the immune response results in the release of an array of cytokines produced by dendritic cells, macrophages, NK cells and epithelial cells $s^{[8-11]}$. Some of these cytokines such as interferons, TNF and IL1 up-regulate the expression of molecules involved in antigen processing and presentation to direct and facilitate the subsequent adaptive immune response ${ }^{[12-}$

${ }^{14]}$. Cytokine regulation of the antigen presentation 
machinery in lung epithelial cells is not well understood. Furthermore, unlike other organs in the body, the respiratory tract is constantly exposed to a variety of airborne pollutants and it is not known if the presence of these pollutants influences the antigen presentation by lung epithelial cells.

In the present study, we examined the expression of class I and class II MHC molecules as well as two other immunologically critical important membrane proteins ICAM 1 (CD54) and B7.1 (CD80) on lung epithelial cells following incubation with, (a) the cytokines IFN $\gamma$, IL1 $\beta$ and TNF $\alpha$, (b) diesel exhaust particles (DEP), and (c) after exposure to both stimuli. Our results indicate a significant basal expression of MHC class I molecules and ICAM 1 on mouse epithelial cells that is further boosted by cytokines. Basal as well as CM induced expression of these markers was significantly inhibited by DEP. Only marginal expression of MHC class II molecules was detected on epithelial cells that was not significantly boosted by cytokines. B7.1 molecule was constitutively expressed on epithelial cells and its expression was not influenced by cytokines or DEP.

\section{MATERIALS AND METHODS}

Cells and reagents: $\mathrm{LA} 4\left(\mathrm{H}-2^{\mathrm{a}}\right.$ expressing $\mathrm{H}-2 \mathrm{~K}^{\mathrm{k}}$ and $\mathrm{H}-2 \mathrm{D}^{\mathrm{d}}$ MHC class I molecules) and TC1 (H-2 $\left.{ }^{\mathrm{b}}\right)$ murine lung epithelial cell lines were obtained from American Type Cell Culture (Rockville, MD). Both cell lines were maintained in RPMI 1640 culture medium supplemented with glutamine $(2 \mathrm{mM})$, HEPES buffer $(25 \mathrm{mM})$, gentamycin $(20 \mu \mathrm{g} / \mathrm{ml})$, and $10 \% \mathrm{~V} / \mathrm{V}$ fetal bovine serum (Hyclone, Logan, UT). Diesel exhaust particles (DEP Standard Reference Material 2975) were purchased from the National Institute of Standards and Technology (NIST), Gaithersburg, MD. Monoclonal antibodies used for flow cytometric staining were procured from BD-Pharmingen, San Diego, CA. These were anti $\mathrm{MHC}$ I $\left(\mathrm{D}^{\mathrm{d}}\right)-\mathrm{PE}$ (clone 34-5-83); MHC I (D $)$ PE (clone KH95); MHC II (IA ${ }^{\mathrm{d}}$ )-FITC (clone 39-10-8); MHC II (IA ${ }^{\mathrm{b}}$ )-FITC (clone AF6-120.1); CD54-FITC (clone 3E2); CD80-PE (clone 16-10A1); FcBlock CD16/32 (clone 2.4G2) and corresponding isotypic controls. Recombinant mouse cytokines IFN $\gamma$, TNF $\alpha$ and IL1 $\beta$ were bought from Cell Sciences, Canton, MA.

In vitro treatment of cells and flow cytometry: A stock suspension of DEP $(5 \mathrm{mg} / \mathrm{ml})$ was prepared in sterile normal saline and sonicated for one minute using a probe sonicator (Microson Ultrasonic Cell Disrupter). Cells were seeded in culture at a cell density of $2 \times 10^{4} / \mathrm{ml}\left(10 \mathrm{ml} /\right.$ culture flask, $25 \mathrm{~cm}^{2}$ area $)$ and after 72 hours, the sonicated DEP suspension and/or cytokines were added at the desired concentration. Four replicate assay wells were set up for each treatment group. After 48 hours, cells were detached by treatment with trypsinEDTA and washed with phosphate buffered saline containing $1 \%$ FCS and $0.01 \%$ sodium azide. Cells $\left(0.5 \times 10^{6}\right)$ in $50 \mu \mathrm{l}$ volume were incubated with $1 \mu \mathrm{g}$ CD16/32 antibody (Fc-Block) in ice for $20 \mathrm{~min}$. One $\mu \mathrm{g}$ each of the required staining antibodies or isotype control antibodies were added and incubation continued for $30 \mathrm{~min}$. Cells were washed once and fixed in ice cold 4\% paraformaldehyde solution in PBS. Cells were analyzed in a BD LSR II Flow cytometer using the Argon-ion $488 \mathrm{~nm}$ laser. BD FACSDiva software was used to analyze the data.

Statistics: Flow cytometric data analysis by using FACSDiva software provided values for mean fluorescence intensity, coefficient of variation $[\mathrm{CV}=$ (SD/mean) x100], and number of events (degrees of freedom) for any data set. By using these values, $p$ values of significance of difference between any two data sets were calculated by using an on-line Student's t-test (http://home.clara.net/sisa/t-test.htm). Only representative results have been shown that demonstrate the effects of cytokine and/or DEP on the expression of various markers.

\section{RESULTS}

Modulation of surface expression of MHC molecules on LA4 lung epithelial cells by cytokines and DEP. Staining of LA4 cells with anti-H-2D ${ }^{\mathrm{d}}$ antibodies indicated that $83 \%$ LA4 cells were positive for MHC I expression (Figure 1). Treatment with cytokine mix $[\mathrm{CM}$, a mixture of each of IFN $\gamma \quad(10 \mathrm{ng} / \mathrm{ml})$, $\operatorname{IL} 1 \beta(10 \mathrm{ng} / \mathrm{ml})$ and $\mathrm{TNF} \alpha(2 \mathrm{ng} / \mathrm{ml})]$ increased the percentage of MHC I +ve cells to $97 \%$.

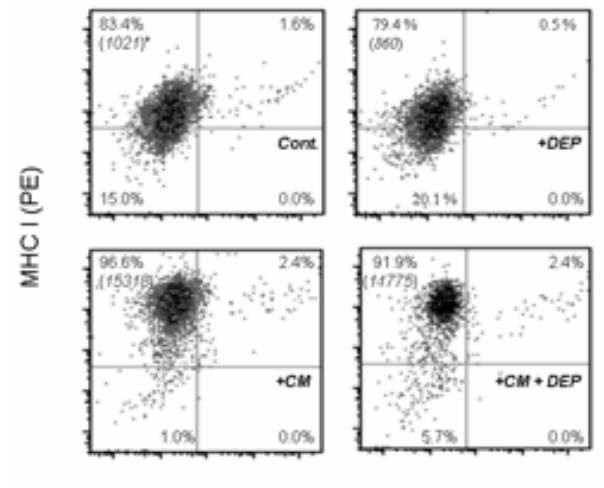

MHC II (FITC)

Fig.1: Expression of MHC I and II on LA4 epithelial cells treated with a cytokine mixture (CM, 10 $\mathrm{ng} / \mathrm{ml}$ each of IFN $\gamma$, and IL $1 \beta$ and $2 \mathrm{ng} / \mathrm{ml}$ of $\mathrm{TNF})$, or DEP $(25 \mu \mathrm{g} / \mathrm{ml})$ or both for 48 hours. Cells were harvested, washed and stained with anti-MHC class I (PE) and anti MHC class II (FITC) antibodies as described in methods. Values in parentheses denote mean channel fluorescence for MHC I expression. 
Since a large percentage of control LA4 cells had a basal expression of MHC I molecules, the effect of CM on the percentage of MHC I +ve cells was small. However a marked effect of $\mathrm{CM}$ on the quality of MHC I expression on LA4 cells was clearly seen from the mean channel of fluorescence (MCF) data that reflects the number of MHC I molecules expressed per cell. MCF of MHC I expression increased 15 fold from 1021 on control LA4 cells to 15318 on CM treated cells (Figure 1). Treatment with $25 \mu \mathrm{g} / \mathrm{ml}$ DEP marginally yet significantly depressed basal MHC I expression on LA4 cells $(p<0.01)$. When LA4 cells were treated with both CM and DEP, a significant (25\%) reduction in the MCF of MHC I expression as compared to high MHC I expression levels on CM treated LA4 cells, occured $(\mathrm{p}<0.01)$. Few LA4 cells expressed MHC

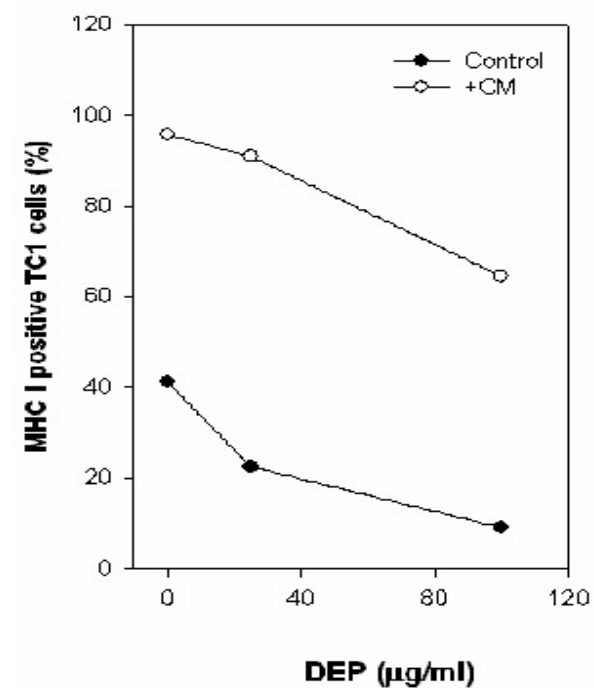

Fig. 2: Expression of MHC I on TC1 epithelial cells treated with a cytokine mixture $(\mathrm{CM}, 10 \mathrm{ng} / \mathrm{ml}$ each of IFN $\gamma$, IL1 $\beta$ and TNF), or DEP ( 25 and $100 \mu \mathrm{g} / \mathrm{ml}$ ) or both for 48 hours. Cells were harvested, washed and stained with anti-MHC class I antibody as described in methods.

II molecules and there was no significant effect of CM on this parameter (Figure 1). For TC1 epithelial cells (MHC H-2 ${ }^{\mathrm{b}}$ ), about $40 \%$ cells had basal MHC I expression that was boosted to almost $100 \%$ by the CM (Figure 2). Results in Figure 2 further show that DEP induces a dose dependent decline in the MHC I expression on both control or CM treated TC1 cells $(\mathrm{p}<0.01)$. Interestingly, while the MHC I positive cells increased significantly as a result of cytokine treatment with cytokines, the average expression of MHC I molecules on TC1 cells was not significantly altered.
The MCF values for DEP $(25 \mu \mathrm{g} / \mathrm{ml})$ treated, cytokine treated and cytokine + DEP treated cells were 621,538 , 875 and 569, respectively. Like LA4 cells however, TC1 cells also had negligible expression of MHC II molecules that could not be significantly boosted by $\mathrm{CM}$ (results not shown).

Modulation of expression of CD54 (ICAM 1) molecules on LA4 epithelial cells by cytokines and DEP. ICAM-1 expressed on antigen presenting cells is required for the activation of $\mathrm{T}$ lymphocytes (11). Results in Figure 3 indicate that very few LA4 cells had a basal expression of ICAM 1, but a significant expression of this molecule was induced by CM ( $\mathrm{p}<0.01)$. A marginal decline in percentage of ICAM 1 positive cells induced in response to CM was seen when DEP was added to the culture. The mean channel of expression of ICAM 1 was however not altered in presence of DEP (Figure 3).

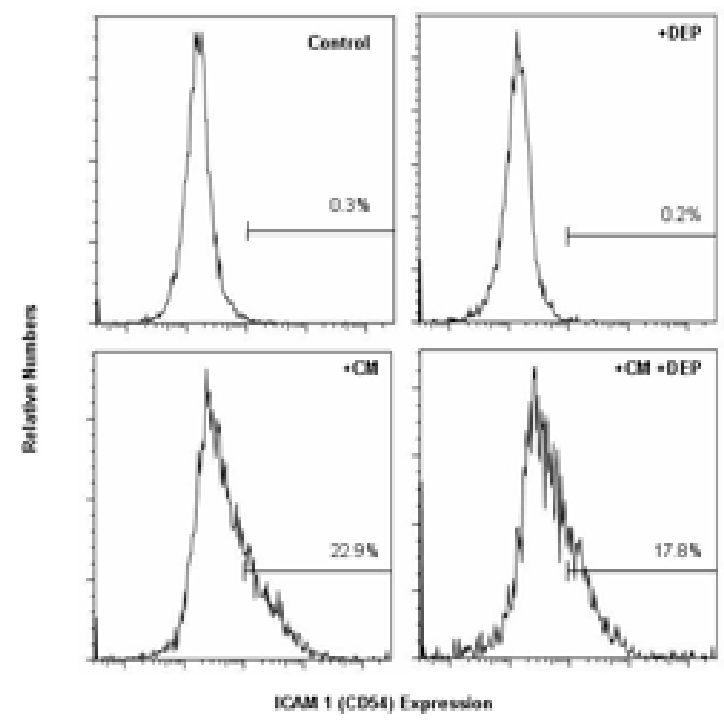

Fig. 3: Expression of ICAM 1 (CD54) on LA4 epithelial cells treated with a cytokine mixture (CM) or DEP $(25 \mu \mathrm{g} / \mathrm{ml})$ or both for 48 hours. Cells were harvested, washed and stained with anti-CD54 antibodies as described in methods. Horizontal lines represents the CD54 expression above the baseline staining with isotypic control.

ICAM 1 (CD54) and B7.1 (CD80) expression on TC1 epithelial cells. Almost all TC1 epithelial cells expressed B7.1 molecules and treatment with $\mathrm{CM}$ or DEP had no effect on the percentage of TC1 cells expressing B7.1 molecule (Figure 4). Mean channel fluorescence for B7.1 staining was 7770, 9853, 7597 and 9022 respectively for control, CM treated, DEP 
treated and $\mathrm{CM}+\mathrm{DEP}$ treated $\mathrm{TC} 1$ cells. These values indicate that the average B7.1 expression per cell were unaltered by $\mathrm{CM}$ or DEP treatment. Basal expression of ICAM $1(20 \%$ cells $+v e)$ was higher in TC1 cells as compared to the LA4 cells that did not express ICAM 1 (Figure 3). CM treatment markedly boosted ICAM 1 expression since $94 \%$ of the CM treated TC1 cells expressed ICAM 1 molecule $(\mathrm{p}<0.01)$. Treatment with DEP $(25 \mu \mathrm{g} / \mathrm{ml})$ reduced the expression of ICAM 1 on control as well as $\mathrm{CM}$ treated $\mathrm{TC1}$ cells by approximately $30 \%(\mathrm{p}<0.001)$.

\section{DISCUSSION}

MHC molecules are required for the development of adaptive immune responses and are also involved in innate immunity. $\mathrm{T}$ cells recognize antigenic peptides in association with $\mathrm{MHC}$ molecules ${ }^{[2]}$. For infectious agents like viruses that grow inside the host cells, viral peptides are presented in association with class I MHC molecules to activate cytotoxic $\mathrm{T}$ cells that eventually destroy the virus infected target cells ${ }^{[1]}$. Dendritic cells and alveolar macrophages are the antigen presenting cells (APCs) in lungs that process and present exogenous antigens (e.g. bacterial antigens) to $\mathrm{T}$ cells for induction of an appropriate adaptive immune response $^{[3,4]}$.

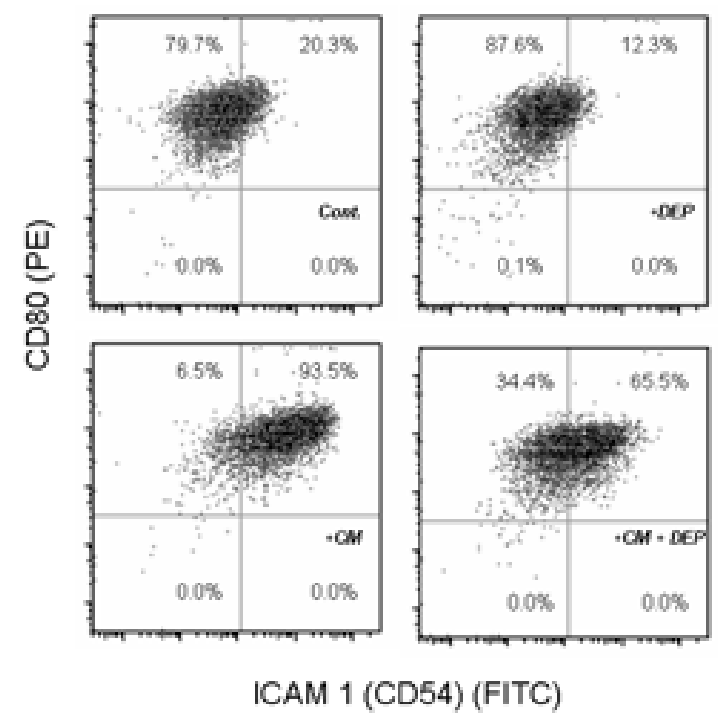

Fig. 4: Expression of B7.1 (CD80) and CD54 on TC1 epithelial cells treated with a cytokine mixture $(\mathrm{CM}, 10 \mathrm{ng} / \mathrm{ml}$ each of IFN $\gamma$, IL1 $\beta$ and TNF), or DEP $(25 \mu \mathrm{g} / \mathrm{ml})$ or both for 48 hours. Cells were harvested, washed and stained with anti-CD80 (PE) and anti CD54 (FITC) antibodies as described in methods.
Interaction of $\mathrm{T}$ cell receptors with $\mathrm{MHC}$ molecules displaying the antigenic peptide imparts antigen specificity to the process of $\mathrm{T}$ cell activation. However these interactions do not by themselves constitute sufficient condition for $\mathrm{T}$ cell activation. Signaling originating through interactions between a variety of other accessory molecules on $T$ cells and antigen presenting cells are needed for $\mathrm{T}$ cells to be activated.

A crucial accessory interaction is between ICAM 1 (CD54) molecule on APCs and the LFA1 (CD11a/CD18) molecules on $\mathrm{T}$ cells ${ }^{[11-13]}$. Additional interactions between the B7 family of molecules on APCs with the CD28 family of molecules on T cells determine whether the $\mathrm{T}$ cells would be activated or tolerized $^{[18,19]}$.

In the present communication, we have studied the basal and cytokine activated expression levels of MHC, ICAM and B7 molecules on established cell lines of lung epithelial cells in culture. Our results indicate that only $40 \%$ and $84 \%$ of unstimulated TC1 and LA4 cells respectively expressed detectable levels of MHC I molecules. Treatment with a mixture of cytokines IFN $\gamma$, IL1 $\beta$ and TNF $\alpha$ (cytokine mixture, CM) markedly boosted the MHC I expression on both cells. The expression of accessory molecule ICAM 1 was simultaneously boosted by $\mathrm{CM}$ in both LA4 and TC1 cells.

Cytokines such as those in the CM (IFN $\gamma, \mathrm{TNF} \alpha$ and IL1 $\beta$ ) are released by many cell types like macrophages, dendritic cells and NK cells, in response to activation of TLRs by components of pathogens ${ }^{[12-}$ 14]. Lung epithelial cells are prime targets of viral infections by agents such as influenza virus and respiratory syncytial virus, and initial activation of MHC I and ICAM 1 expression by CM may prepare the epithelial cells to better present viral antigens to $\mathrm{T}$ cells. B7.1 (CD80) is another molecule that is involved in the $\mathrm{T}$ cell APC interaction and $\mathrm{TC1}$ cells had a high constitutive expression of B7.1.

We have recently shown that lung epithelial cells can take up substantial amounts of DEP in culture ${ }^{[20]}$, and this may influence various cellular processes. Results of this present study indicate that DEP treatment depressed basal as well as CM induced expression of MHC I and ICAM 1 expression. No effect of DEP was however found on B7.1 expression, however. These results suggest that DEP did not cause a nonspecific decline in expression of membrane markers but rather some specific markers were selectively depressed. Suppression of MHC I and ICAM 1 expression by DEP may result in less efficient presentation of viral antigens by DEP exposed lung 
epithelial cells. Exposure to DEP is known to result in predisposition to asthma ${ }^{[21-23]}$ as well as susceptibility to viral and bacterial infections ${ }^{[24-26]}$. An altered antigen presentation by DEP exposed epithelial cells may be a contributory factor in these predispositions.

Epithelial cells had only a marginal expression of MHC class II molecules, which are involved in presentation of exogenous antigens like bacterial antigens to helper $\mathrm{T}$ cells. Low expression of MHC class II molecules on lung epithelial cells likely signifies their low involvement in the presentation of bacterial antigens. It should however be pointed out that in lungs epithelial cells outnumber alveolar macrophages by many orders of magnitude. Therefore even if a small fraction of epithelial cells express MHC II molecules and presented bacterial antigens, that may become a significant factor in the overall generation of antibacterial immune response in lungs.

In conclusion, we have demonstrated that mouse lung epithelial cells express many necessary markers required for antigen presentation, for MHC I pathway. Expression of antigen presentation markers is boosted by inflammatory cytokines while DEP exposure suppresses both basal and cytokine induced markers involved in antigen presentation. Exposure to DEP may thus potentially interfere with development of specific immune responses in lungs and lead to a suboptimal adaptive immune response to airborne pathogens.

\section{ACKNOWLEDGMENT}

RKS was supported by an NRC senior fellowship.

\section{REFERENCES}

1. Germain, R.N. and D. H. Margulies. 1993. The Biochemistry and Cell Biology of Antigen Processing and Presentation. Ann Rev Immunol., 11: 403-450

2. van der Merwe, P.A. and S. J. Davis, 2003. Molecular interactions mediating $\mathrm{T}$ cell antigen recognition. Ann. Rev. Immunol., 21: 659-684

3. Guermonprez,P., J. Valladeau, L. Zitvogel, C. héry and S. Amigorena, 1997. Antigen presentation and $\mathrm{T}$ cell stimulation by dendritic cells. Ann. Rev. Immunol. 20: 621-667

4. Watts, C. 1997. Capture and processing of exogenous antigens for presentation on MHC molecules. Ann. Rev. Immunol., 15: 821-850

5. Jamaluddin, M., S. Wang, R.P. Garofalo, T. Elliott, A. Casola, S. Baron, A.R.Brasier, 2001. IFN-beta mediates coordinate expression of antigenprocessing genes in RSV-infected pulmonary epithelial cells, Am J Physiol Lung Cell Mol Physiol. 280:L248-57
6. Rimmelzwaan, G.F., A.C. Boon, M.M. GeelhoedMieras, J.T. Voeten, R.R. Fouchier and A.D. Osterhaus, 2004. Human airway epithelial cells present antigen to influenza virus-specific CD8+ CTL inefficiently after incubation with viral protein together with ISCOMATRIX.. Vaccine. 22:2769-75

7. Debbabi, H., S. Ghosh, A.B. Kamath, J. Alt, D.E. Demello, S. Dunsmore, S.M. Behar, 2005. Primary type II alveolar epithelial cells present microbial antigens to antigen-specific CD4+ $\mathrm{T}$ cells.Am $\mathrm{J}$ Physiol Lung Cell Mol Physiol., 289: 274-9

8. Bals, R. and P.S. Hiemstra 2004.Innate immunity in the lung: how epithelial cells fight against respiratory pathogens. Eur Respir J. 23: 327-33.

9. Gordon, S.B. and R.C. Read 2002. Macrophage defenses against respiratory tract infections.. $\mathrm{Br}$ Med Bull. 61: 45-61

10. Krutzik, S.R. and R.L. Modlin 2004. The role of Toll-like receptors in combating mycobacteria. Semin Immunol. $16: 35-41$

11. Junqueira-Kipnis, A.P., A. Kipnis, A. Jamieson, M.G. Juarrero, A. Diefenbach, D.H. Raulet, J. Turner and I. Orme 2003. NK cells respond to pulmonary infection with Mycobacterium tuberculosis, but play a minimal role in protection. J Immunol. 171: 6039-45

12. Dabbagh, K. and D.B.Lewis, 2003. Toll-like receptors and T-helper-1/T-helper-2 responses. Curr Opin Infect Dis. 16:199-204

13. Eriksson, M., S.K. Meadows, S. Basu, T.F. Mselle, C.R. Wira, C.L. Sentman, 2006. TLRs mediate IFN-gamma production by human uterine NK cells in endometrium.J Immunol. 176:6219-24

14. Fricke, I., D. Mitchell, J. Mittelstadt, N. Lehan, H. Heine, T. Goldmann, A. Bohle, S. Brandau, 2006. Mycobacteria induce IFN-gamma production in human dendritic cells via triggering of TLR2.J Immunol.176:5173-82

15. Lebedeva, T., M.L. Dustin and Y. Sykulev, 2005. ICAM-1 co-stimulates target cells to facilitate antigen presentation. Curr Opin Immunol. 17:251-8

16. Ghosh, S. and R.K. Saxena, 2004. Early effect of Mycobacterium tuberculosis infection on Mac-1 and ICAM-1 expression on mouse peritoneal macrophages, Exp Mol Med. 36:387-95

17. Stanciu, L.A. and R. Djukanovic, 1998. The role of ICAM-1 on T-cells in the pathogenesis of asthma.Eur Respir J., 11:949-57

18. Wang, S. and L. Chen, 2004. T lymphocyte cosignaling pathways of the B7-CD28 family.Cell Mol Immunol. 1:37-42 
19. Greenwald, R.J., G.I. Freeman and A.H. Sharpe, 2005. The B7 family revisited. Annu Rev Immunol. 23:515-48

20. Saxena, R.K., M.I. Gilmour and M. Hays, 2006. A novel method for the quantitative estimation of diesel exhaust particle uptake by lung epithelial cells and macrophages. (Submitted)

21. D'Amato, G., G. Liccardi, M. D'Amato and M. Cazzola, 2002. Respiratory allergic diseases induced by outdoor air pollution in urban areas. Monaldi Arch Chest Dis. 57:161-3

22. Diaz-Sanchez, D., L. Proietti and R. Polosa, 2003. Diesel fumes and the rising prevalence of atopy: an urban legend? Current Allergy Asthma Rep. 3:14652

23. Gilmour, M.I., T. Stevens and R.K. Saxena, 2006. Effect of Particles on the Immune System. In Particle Toxicology (eds. K. Donaldson and P. Borm) CRC Press, Boca raton, FL. USA. (In Press)
24. Siegel, P.D., R.K. Saxena, Q.B.Saxena, J.K. Ma, J.Y. Ma, X.J. Yin, V. Castranova, N. Al-Humadi, D.M. Lewis, 2004. Effect of diesel exhaust particulate (DEP) on immune responses: contributions of particulate versus organic soluble components, J. Toxicol Environ Health A. 67:22131

25. Saxena, R.K., Q.B. Saxena, D.N. Weissman, J.P. Simpson, T.A. Bledsoe and D.M. Lewis, 2003. Effect of diesel exhaust particulate on bacillus Calmette-Guerin lung infection in mice and attendant changes in lung interstitial lymphoid subpopulations and IFN gamma response. Toxicol Sci., 73:66-71

26. Ito, T., H. Okumura, N. Tsukue, T. Kobayashi, K. Honda and K. Sekizawa, 2006 Effect of diesel exhaust particles on mRNA expression of viral and bacterial receptors in rat lung epithelial L2 cells. Toxicol Lett. 5: 66-70. 